\title{
Les disparités régionales des activités tertiaires, nouvel enjeu de la politique régionale
}

\section{Introduction}

Le développement de l'emploi et des activités tertiaires dans les sociétés post-industrielles constitue une tendance inscrite dans le long terme. Trois explications peuvent être données de cette expansion du tertiaire (v. FUCHS, 1968; D. BELL, 1973; OCDE, 1977): l'évolution intervenue dans les valeurs et les modes de vie des individus a fait exploser la demande de services personnels; la dernière vague d'innovation technologique a provoqué une incitation à appliquer les progrès scientifiques dans le secteur tertiaire et en particulier dans les activités de service souvent incorporées au secteur industriel (gestion, études de marché, recherche, contrôle de fabrication, etc.). C'est tout le sens de la révolution informatique en cours. Enfin, la division intense du travail et la complexité croissante de l'économie ont fait émerger une demande jusqu'ici latente de service aux entreprises. Le développement de ces nouvelles activités de services à la production apparaît comme un phénomène fondamental, caractérisant un changement proprement qualitatif des systèmes productifs modernes.

Une nouvelle étape semble franchie dans le développement de nos sociétés quand leur dynamique spatio-économique n'a plus comme unique moteur la production mécanique de biens matériels. Certes, les services ne vont pas remplacer l'industrie et l'agriculture, mais à l'avenir l'interaction entre la production de biens et la production de services sera sans doute plus intense. Les années 70 marquent probablement un tournant vers un nouveau mode de développement économique où l'organisation des systèmes productifs intègre horizontalement la production industrielle et agricole aux activités de service. Les frontières entre les trois secteurs économiques deviennent floues et mouvantes.

Depuis que A. FISCHER (1947) et C. CLARK (1960) ont proposé leur fameuse division de l'économie en trois secteurs le contenu même de la notion de «tertiaire» a changé. La croissance et la diversification du tertiaire, le rôle des nouvelles activités de service dans le système productif renouvellent la problématique géographique des localisations tertiaires.

L'objectif de cet article est double. D'une part, il se propose de décrire la distribution spatiale des activités de service à l'échelle des cantons et des villes suisses. D'autre part, il apporte quelques réflexions sur la nécessité de poser d'une autre manière la question de l'intégration des activités de service dans les politiques de développement régional. La situation actuelle assure désormais un rôle primordial aux activités de service aux entreprises sur le plan de l'animation régionale. Une politique régionale cohérente de valorisation des régions périphériques doit par conséquent stimuler l'offre régionale de ces services qui semblent si décisifs pour l'accroissement du potentiel de développement endogène des systèmes productifs régionaux.

\section{La répartition intercantonale de l'emploi tertiaire: les spécificités fonctionnelles du centre et de la périphérie}

La répartition intercantonale de l'emploi tertiaire a été décrite de manière approfondie dans une intéressante étude du SCRIS (1978) mais cette étude demande à être renouvelée et complétée. Deux points essentiels doivent être reexaminés:

- d'une part, la nouvelle typologie des activités tertiaires proposée par Browning et Singelmann (1978) permet de mieux rendre compte de manière synthétique des spécialisations et des associations d'activités qui organisent l'espace économique helvétique;

- d'autre part, la structure intercantonale des activités est ici envisagée sous l'angle de la problématique centre-périphérie.

Notre étude livre une image hiérarchisée de la localisation des activités tertiaires: concentration des services de commandement et de gestion économique dans quelques cantons-centres, diffusion des services banalisés dans les régions périphériques; polarisation des emplois qualifiés et à forte valeur ajoutée dans le centre, diffusion des emplois peu qualifiés et à faible valeur ajoutée en direction de la périphérie. En raison de leurs profils de spécialisa-

Antonio Cunha, Laurent Bridel, Prof. Dr., Institut de géographie, Université de Lausanne, Dorigny, 1015 Lausanne 
tion et de la diversité des services qu'ils accueillent, les cantons-centres gardent une plus large capacité d'innovation et de développement autonome.

\subsection{La typologie des activités tertiaires de Browning-Singelmann}

A mesure que le secteur tertiaire a crû relativement en importance et s'est diversifié, il a fait naître de nouveaux sujets de réflexion dont l'analyse exige une typologie différente. Depuis les années 60 , de nombreux auteurs ont souligné l'insuffisance des typologies classiques (J.HAUTREUX, 1964; M. LENGELLE, 1966; M. ROCHEFORT, 1976; P. GEORGE, 1978; A. BAILLY: D. MAILLAT, 1986).

Dans une communication récente A. BARCET (1986) a dressé un tableau critique des nombreuses typologies élaborées au cours des dernières années sur lequelle il nous semble inutile de revenir ici. Il apparaît clairement que toute classification est un cons- truit, une simple convention qui cherche à récupérer le concret. La multiplication des typologies répond à de nouvelles exigences de recherche, elle est aussi le résultat de la pratique de l'infirmationnisme naif, pratique selon laquelle il suffirait de découvrir un exemple incompatible avec le schéma adopté pour le rendre inopérant.

Quoiqu'il en soit, la géographie des activités tertiaires ne peut esquiver le problème de l'extrême hétérogénéité des activités de service. Il est nécessaire d'essayer de dégager des types d'activités de manière à mieux préciser la relation possible entre leurs localisations et la dynamique spatio-économique. Pour qu'un mode particulier de classification conduise à une interprétation de la réalité géographique, il faut qu'il soit fondé sur des hypothèses relatives à la manière dont les activités tertiaires sont distribuées dans l'espace. Toute typologie doit répondre à des objectifs théoriquement fondés, sans quoi il n'y a qu'accumulation desordonnée d'images parcellaires d'une réalité toujours mouvante.

Tab. 1 Typologie des Services

\begin{tabular}{|c|c|c|c|c|c|c|}
\hline TYPE & $\begin{array}{l}\text { FONCTIONS ECONOMIQUES } \\
\text { PRINCIPALES }\end{array}$ & $\begin{array}{l}\text { DESTINATAIRE } \\
\text { PRINCIPAL }\end{array}$ & $\begin{array}{l}\text { PLACE DANS LE } \\
\text { SYSTEME ECONOM. }\end{array}$ & $\begin{array}{l}\text { NIVEAU DE QUA- } \\
\text { LIFICATION } \\
\text { DU TRAVAIL }\end{array}$ & $\begin{array}{l}\text { VALEUR AJOUTEE } \\
\text { PAR PERSONNE } \\
\text { OCCUPEE }\end{array}$ & $\begin{array}{l}\text { FORME DE PRO - } \\
\text { DUCTION DOMI - } \\
\text { NANTE }\end{array}$ \\
\hline $\begin{array}{l}\text { SERVICES AUX ENTREPRISES } \\
\text { Banques et services fin. } \\
\text { Assurances } \\
\text { Imnobiller } \\
\text { Fiducialres } \\
\text { Bureaux de consell jur1. } \\
\text { Traltement de donnees } \\
\text { Divers }\end{array}$ & $\begin{array}{l}\text { - Satisfaire la demande } \\
\text { d'information des entrep. } \\
\text { - Creer, gerer, stocker et } \\
\text { traiter de l'information } \\
\text { - Stimuler l'innovation }\end{array}$ & Entreprises & $\begin{array}{l}\text { Services } \\
\text { intermediaires }\end{array}$ & Elevé & Elevee & $\begin{array}{l}\text { Privee / } \\
\text { Grandes } \\
\text { entreprises }\end{array}$ \\
\hline $\begin{array}{l}\text { SERVICES DE DISTRIBUTION } \\
\text { Transport et entreposage } \\
\text { Conmunication } \\
\text { Poste } \\
\text { Commerce de detall (non } \\
\text { compris la restauration) }\end{array}$ & $\begin{array}{l}\text { - Satisfaire la demande de } \\
\text { mobilite et de communi- } \\
\text { cation } \\
\text { - Distrivuer les biens, } \\
\text { faire circuler les per- } \\
\text { sonnes et les informat. } \\
\text { - Articuler les activites } \\
\text { de production et de con- } \\
\text { sommation }\end{array}$ & $\begin{array}{l}\text { Mixte (entre- } \\
\text { prises et con- } \\
\text { sommateurs) }\end{array}$ & $\begin{array}{l}\text { Services } \\
\text { Intermedlalres }\end{array}$ & $\begin{array}{l}\text { Moyen / } \\
\text { Heterogene }\end{array}$ & Moyenne & $\begin{array}{l}\text { Privee / } \\
\text { Petites et } \\
\text { grandes } \\
\text { entreprises }\end{array}$ \\
\hline $\begin{array}{l}\text { SERVICES SOCIAUX } \\
\text { Services medicaux } \\
\text { HODitaux } \\
\text { Education } \\
\text { Administartion publique } \\
\text { Services sociaux et pro- } \\
\text { fessionnels divers }\end{array}$ & $\begin{array}{l}\text { - Satisfaire la demande de } \\
\text { services collectifs } \\
\text { - Ameliorer les conditions } \\
\text { d'existence collectives } \\
\text { - Reguler et controler le } \\
\text { fonctionnement du systé- } \\
\text { me socio-economique }\end{array}$ & $\begin{array}{l}\text { Mixte (consom- } \\
\text { mateurs et } \\
\text { entreprises) }\end{array}$ & $\begin{array}{l}\text { Services } \\
\text { finaux }\end{array}$ & $\begin{array}{l}\text { Moyen / } \\
\text { Heterogene }\end{array}$ & Moyenne & $\begin{array}{l}\text { Publ1c / } \\
\text { Pet1tes et } \\
\text { grandes } \\
\text { entreprises }\end{array}$ \\
\hline $\begin{array}{l}\text { SERVICES PERSONNELS } \\
\text { Services domestlaues } \\
\text { Hotels et hebergement } \\
\text { Restaurat Ion } \\
\text { Services de reparation } \\
\text { Blanchisserle, nettoyage } \\
\text { Colffeur, solon de beaute } \\
\text { Lolsirs et culture }\end{array}$ & $\begin{array}{l}\text { - Satisfaire la demande de } \\
\text { services individuels } \\
\text { - Ameliorer les conditions } \\
\text { d'existence individuelles } \\
\text { - Reproduire le statut in- } \\
\text { dividuel et social }\end{array}$ & Consommateurs & $\begin{array}{l}\text { Service } \\
\text { finaux }\end{array}$ & Faible & Falble & $\begin{array}{l}\text { Privee / } \\
\text { Pet1tes } \\
\text { entreprises }\end{array}$ \\
\hline
\end{tabular}




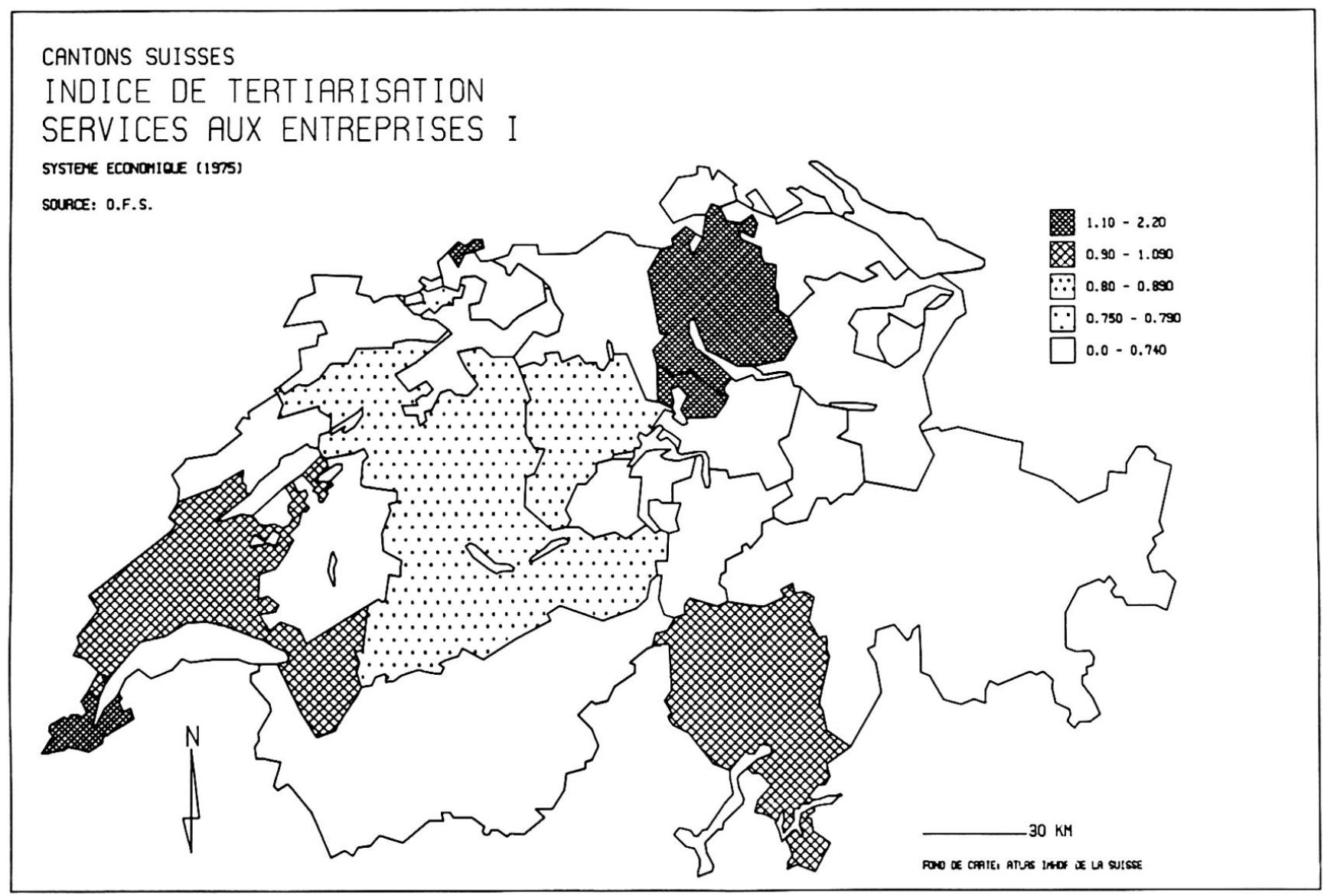

Fig. 1

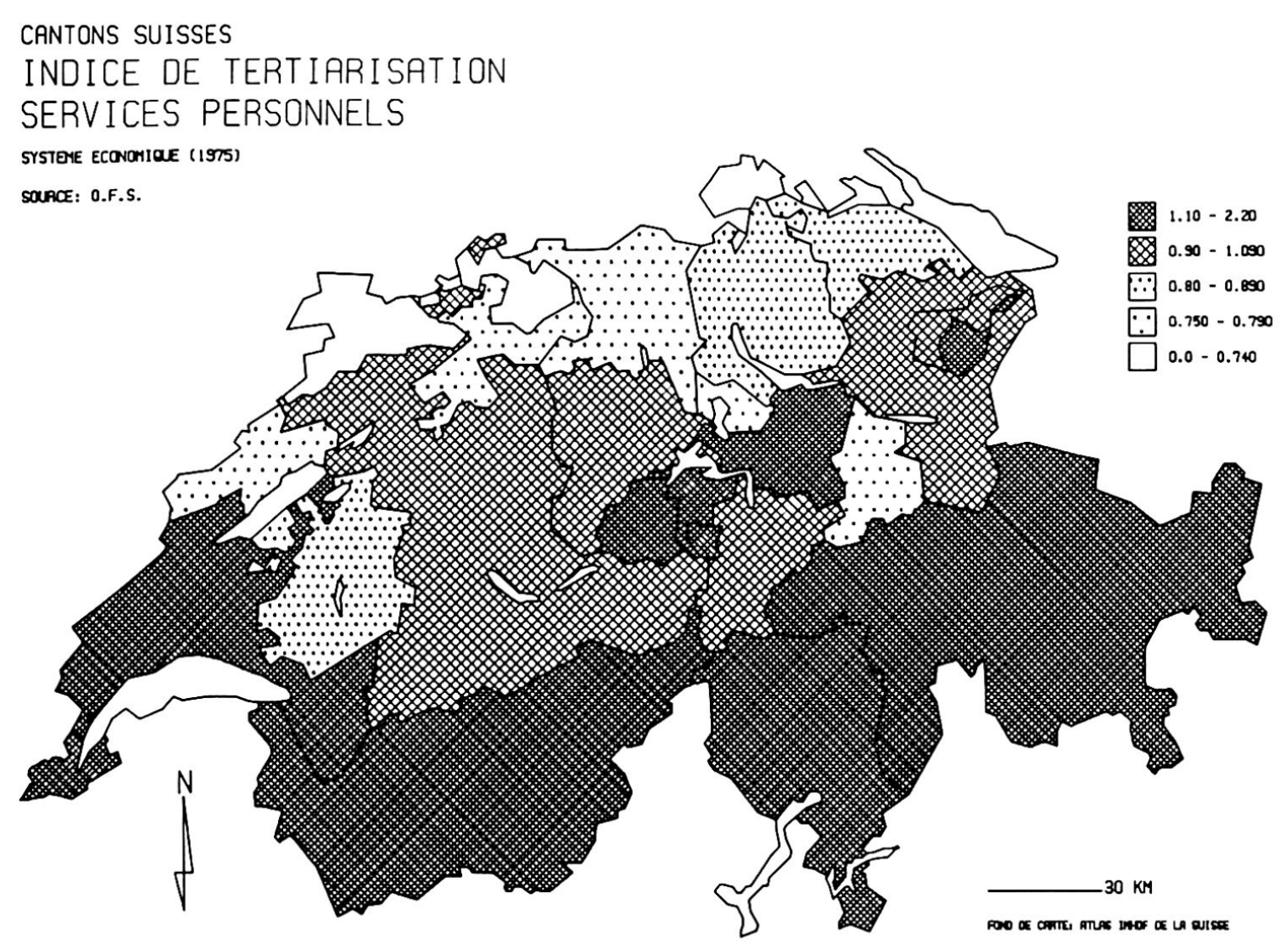

Fig. 2 


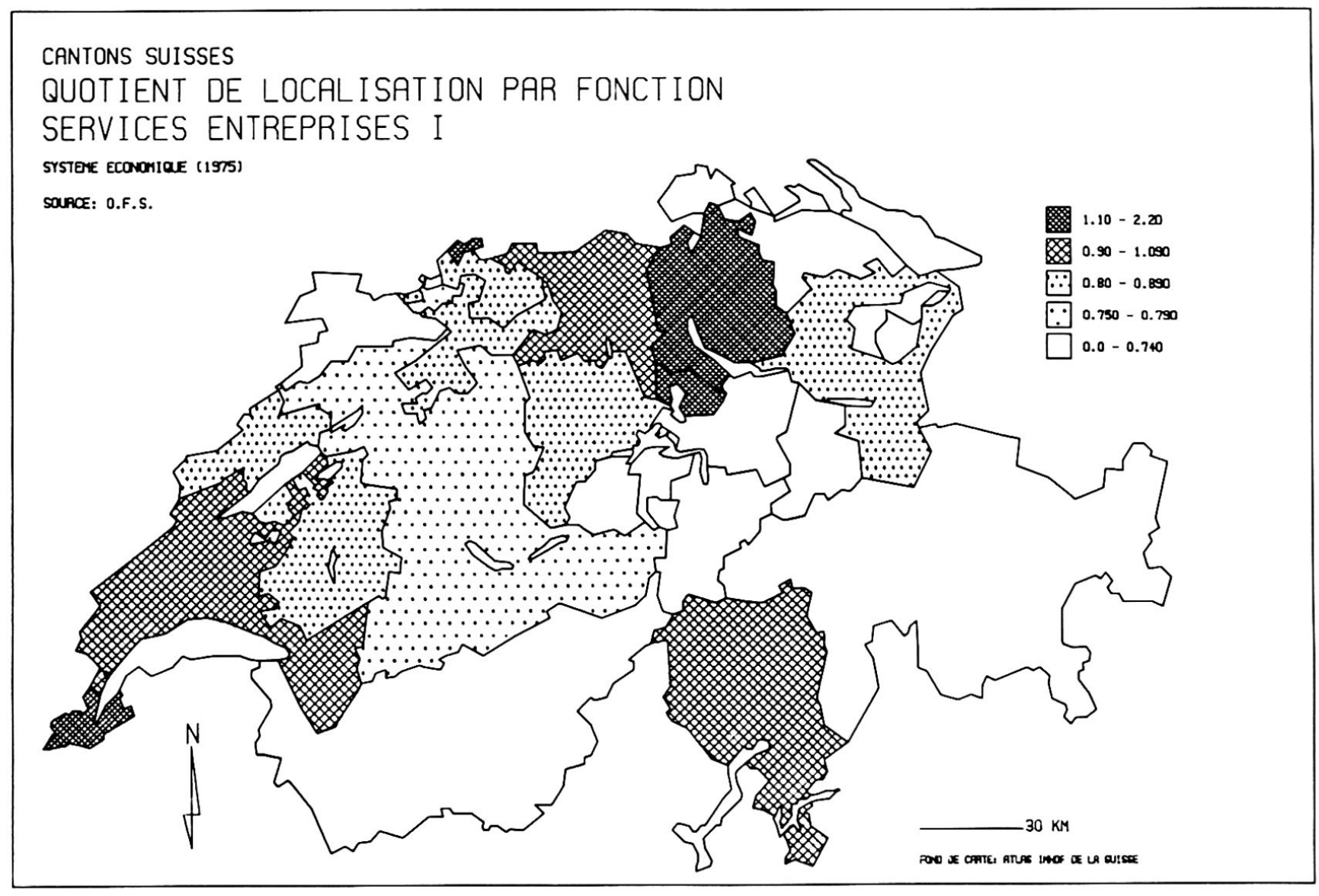

Fig. 3

CANTONS SUISSES

QUOTIENT DE TERTIARISATION SERVICES PERSONNELS STSTEe ECONOHIaE (19t5)

SaACE: O.F.S.

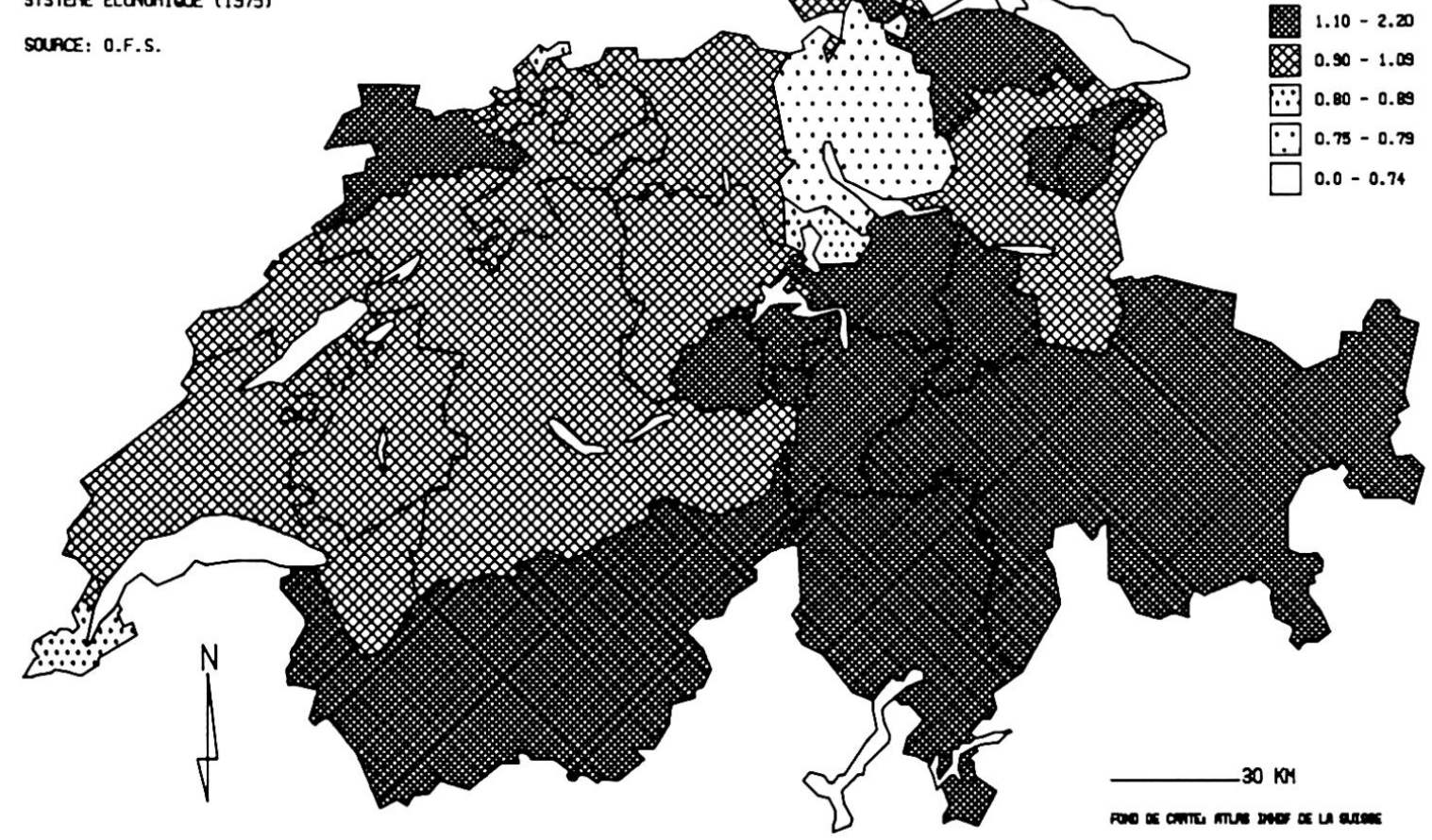

Fig. 4 
L'avantage de la typologie de Browning-Singelmann tient justement au fait qu'elle permet de désigner explicitement un ensemble de critères de discrimination qui autorise une lecture des localisations tertiaires dans les termes de la problématique centre-périphérie, tout en conservant l'accent sur l'aspect économique de ces activités.

Le tableau 1 résume l'ensemble des critères mobilisés.

En ce qui concerne la Suisse, J. DEISs (1985) montre bien que les services aux entreprises sont les plus dynamiques. Au cours du dernier quart de siècle le poids relatif de ce groupe de branches à l'intérieur du tertiaire s'est considérablement accru (17\% en 1984 au lieu de $11 \%$ en 1960). D'une manière générale, écrit-il, la valeur ajoutée par personne est nettement supérieure à la moyenne dans les services aux entreprises, supérieure à la moyenne dans les services de distribution, inférieure à la moyenne dans les services sociaux et très faible dans les services personnels. Tandis que les activités de service personnels occupent en général une main-d'œuvre peu qualifiée, les services à la production font appel à un travail qualifié.

L'étude des caractéristiques des différents types de services nous amène à penser que le développement des services de décision et de gestion économique, dynamiques et hautement productifs, dans certaines régions du pays accentue le déséquilibre tradi- tionnel entre espaces centraux et espaces périphériques. Une politique de développement régional uniquement fondée sur les activités banales et les services publics pourrait hypothéquer le développement à long terme des régions périphériques.

Pour esquisser en termes schématiques les effets d'une croissance du tertiaire sur le territoire nous nous appuierons ici sur l'opposition qui distingue les activités de services aux entreprises des activités de service aux consommateurs. Notre analyse demeure générale au moins à deux points de vue. D'une part, elle ne tient pas compte des modifications parfois spectaculaires à l'intérieur des grandes catégories définies. D'autre part, notre unité de référence spatiale étant ici le canton, nous ne rendons pas compte des disparités intracantonales parfois encore plus marquées que celles constatées entre les cantons.

\subsection{La structure fonctionnelle de l'espace économique:-l'opposition centre-périphérie à l'echelle cantonale}

La typologie des cantons établie en fonction de la répartition de l'activité tertiaire dans les quatre groupes de branches (cf. tableau 1) permet de saisir de manière synthétique les principales oppositions territoriales dans le domaine du tertiaire (figures 1 , 2, 3, 4, sur la base du recensement 1975).

\begin{tabular}{|l|c|c|c|c|c|c|}
\hline & \multirow{2}{*}{$\begin{array}{c}\text { Indice global de } \\
\text { tertiarisation }\end{array}$} & $\begin{array}{c}\text { Services } \\
\text { entreprises I }\end{array}$ & $\begin{array}{c}\text { Services* } \\
\text { entreprises II }\end{array}$ & $\begin{array}{c}\text { Services } \\
\text { distribution }\end{array}$ & $\begin{array}{c}\text { Services } \\
\text { sociaux }\end{array}$ & $\begin{array}{c}\text { Services } \\
\text { personnels }\end{array}$ \\
\cline { 3 - 7 } & 1,18 & 1,48 & 1,45 & 1,18 & 1,08 & 0,98 \\
\hline Centre & 0,96 & 0,79 & 0,79 & 1,02 & 1,04 & 1,00 \\
\hline Sentre moyen & 1,08 & 0,64 & 0,68 & 0,99 & 1,01 & 1,86 \\
\hline Semi-Périp. I & 0,74 & 0,63 & 0,68 & 0,78 & 0,79 & 0,76 \\
\hline Périphérie & 0,73 & 0,35 & 0,36 & 0,71 & 0,87 & 1,07 \\
\hline
\end{tabular}

\begin{tabular}{|l|c|c|c|c|c|c|}
\hline & \multicolumn{5}{|c|}{ Quotient de localisation par fonction } & Services \\
\cline { 2 - 7 } & $\begin{array}{c}\text { Services } \\
\text { entreprises I }\end{array}$ & $\begin{array}{c}\text { Services* } \\
\text { entreprises II }\end{array}$ & $\begin{array}{c}\text { Services } \\
\text { distribution }\end{array}$ & $\begin{array}{c}\text { Services } \\
\text { sociaux }\end{array}$ & $\begin{array}{c}\text { Sere global de } \\
\text { personnels } \\
\text { diversification }\end{array}$ \\
\hline Centre & 1,26 & 1,23 & 0,96 & 0,92 & 0,83 & 3,64 \\
\hline Centre moyen & 0,82 & 0,82 & 1,06 & 1,08 & 1,03 & 3,66 \\
\hline Semi-Périp. I & 0,59 & 0,63 & 1,05 & 0,93 & 1,72 & 3,69 \\
\hline Semi-Périp. II & 0,85 & 0,92 & 0,92 & 1,07 & 1,03 & 4,17 \\
\hline Périphérie & 0,48 & 0,49 & 0,97 & 1,19 & 1,47 & 4,24 \\
\hline
\end{tabular}

* Services aux entreprises II = Services entreprises I - Banques et asssurances 
Les indices de tertiarisation indiquent le rapport entre le pourcentage de l'emploi des branches mentionnées dans la population active totale du canton et ce même pourcentage pour l'ensemble du pays. L'indice situe chaque canton par rapports aux autres et à l'ensemble de la Suisse dont l'indice vaut 1. L'indice est supérieure à 1 si l'emploi tertiaire dans tel ou tel groupe de branches est sur-représenté dans le canton par rapport à la part de la population active de ce canton. Il est inférieure à 1 dans le cas contraire.

Seuls six cantons ont un indice de tertiarisation supérieur ou égal à la moyenne suisse: Zurich, Genève, Bâle-Ville et Vaud, tout d'abord: Zoug également, qui a connu la plus forte croissance de l'emploi tertiaire entre 1955-75 et qui bénéficie d'une législation fiscale qui encourage l'implantation de nouveaux établissements économiques; le Tessin enfin qui doit sa position au rôle bien connu de place financière de la région Lugano-Chiasso et à la forte demande étrangère en consultations juridiques, en particulier dans le domaine de l'immobilier.

Les 20 autres cantons sont nettement sous-représentés. Ce sont les services aux entreprises qui sont les plus fortement concentrés dans un petit nombre de cantons, tandis que les services de distribution et les services sociaux qui s'adressent à une très large clientèle sont mieux répartis dans l'espace. En revanche les services peronnels (cf. figure 2) apparaissent largement sur-représentés dans les périphéries touristiques alpines.

Dans le tableau 2 les cantons ont été classés en 5 groupes au terme d'une analyse de groupement (algorithme de Ward):

Centre: Zurich, Zoug, Genève, Vaud, Bâle-Ville

Centre moyen: Berne, Lucerne, St. Gall, Tessin, Fribourg

Semi-périphérie I: Grisons, Valais, Nidwald

Semi-périphérie II: Soleure, Schaffhouse, Thurgo- vie, Neuchâtel, Bâle-Campagne, Argovie

Périphérie: Uri, Glaris, Schwytz, Appenzell-Ext., Appenzell-I., Obwald, Jura

Tous les indices de tertiarisation décroissent du centre vers la périphérie à l'exception des services personnels dont l'indice est particulièrement élevé dans les cantons périphériques à «vocation touristique». La lecture en ligne du tableau est également intéressante: dans les cantons-centres le poids du tertiaire par fonction diminue presque linéairement quand on passe des services aux entreprises aux services plus banalisés et à faible niveau de qualification. Dans les cantons périphériques on dégage la tendance inverse.

L'analyse des quotients de localisation ne fait que renforcer ce constat. Ces quotients de localisation (tableau 3) peuvent être interprétés comme des mesures du degré de spécialisation des cantons dans les différentes branches tertiaires. La matrice ordonnable (cf. figure 5) nous donne une hiérarchie des niveaux de spécialisation tertiaire et permet de restituer de manière plus détaillée les caractéristiques fonctionnelles des systèmes productifs cantonaux. Les groupes de cantons signalés précédemment sont clairement caractérisés par les valeurs des quotients. Le groupe des cantons-centres présente de fortes valeurs pour les branches de services aux entreprises, témoignant d'une spécialisation axée sur les services plus qualifiés et à plus forte valeur ajoutée, tandis que les cantons périphériques accueillent les services banaux principalement destinés aux consommateurs. L'image qui se dégage des cartes 3 et 4 et des tableaux 2 et 3 (services sociaux et de distribution) révèle une structure des spécialisations fonctionnelles des cantons tout à fait évidente: on peut conclure que les cantons-centres bénéficient outre la localisation de services généraux diversifiés (une faible valeur de l'indice de diversification indique une forte diversification) de la concen-

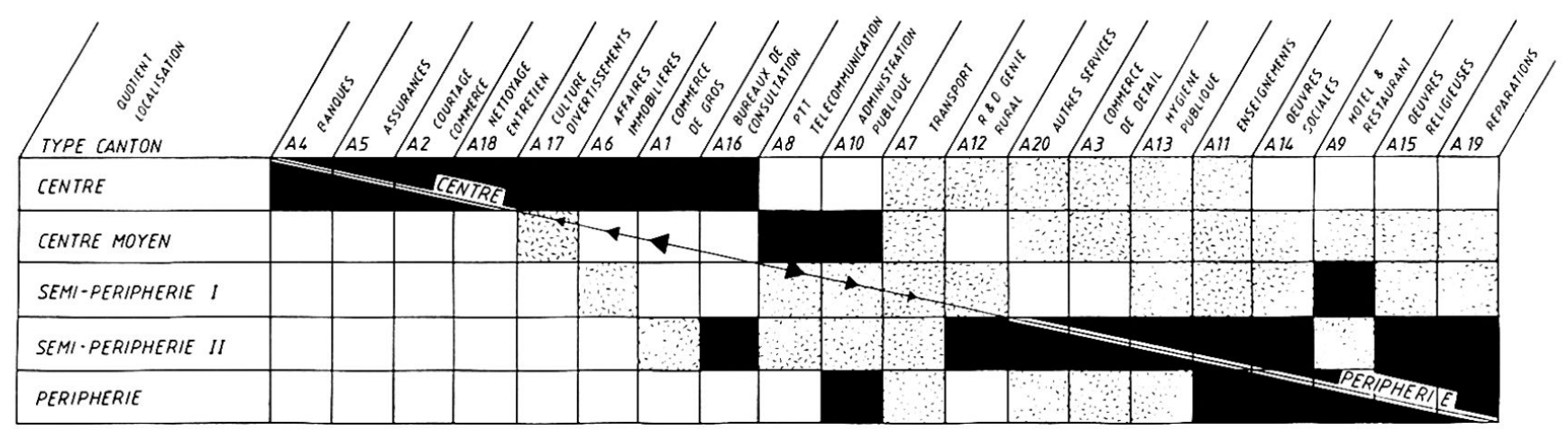

Fig. 5 Matrice ordonnable des quotients de localisation des branches du tertiaire suisse en 1975 
tration de services à la production dont ont sait qu'ils jouent un rôle stratégique dans le développement économique à long terme.

Les disparités spatiales qu'on relève dans la distribution de l'emploi tertiaire sont un aspect important du problème régional dans les sociétés post-industrielles. Cependant, il nous semble indispensable de sortir du découpage cantonal pour décrire au moins sommairement les enjeux territoriaux du développement du tertiaire.

\section{La distribution de l'emploi tertiaire à l'échelle du système urbain}

La multitude des contacts et des échanges d'information et de services que requiert la société post-industrielle s'effectue dans un système de lieux centraux. L'organisation territoriale de l'économie moderne repose sur ces points forts que sont les villes. Le parallélisme entre hiérarchie dimensionnelle des villes et hiérarchie des niveaux de service est un principe général que la théorie géographique et l'observation empirique ont fait admettre mais qui n'en appelle pas moins vérification. L'analyse menée à l'échelle du réseau urbain montre que la part des services aux entreprises augmente régulièrement avec la taille des ville. Cependant, la relation est inégalement applicable aux diverses activités tertiaires. La concentration de certaines fonctions supérieures au sommet de la hiérarchie urbaine laisserait supposer que les villes moyennes et petites ne seraient pas à même d'exercer dans ce domaine le rôle de relais que la théorie leur attribue et que de ce fait elles se montreraient incapables de générer les économies externes nécessaires à un développement cumulatif de leur zone d'influence (A. CUNHA. J.-B. RACINE, 1984). Notre propos sera ici de montrer la pertinence de cette représentation d'ensemble. Deux séries de résultats intéréssants ont été obtenus à partir des données disponibles à cette date (Recensement fédéral des entreprises, 1975).

\subsection{La verticalité du réseau urbain}

La notion de verticalité du réseau urbain s'exprime facilement à travers l'application de la loi rang-taille, utilisée ici "en coupe» de manière à faciliter les comparaisons directes, de service à service, de la distribution de l'emploi tertiaire dans le réseau urbain. En reportant les droites de régression à une origine commune, il est possible de visualiser clairement le phénomène (cf. figure 6). Une forte pente traduit simplement un degré élevé de concentration de l'emploi dans les niveaux supérieurs de la hiérarchie urbaine. L'analyse met en évidence la forte con- centration de certains services spécialisés (traitement de données, publicité, études de marché, assurances, conseils juridiques) au sommet de la pyramide des lieux centraux. A l'inverse, la distribution des services résidentiels, ici représentés par une variable «témoin», le commerce de détail, suit plutôt la loi de répartion de la population active, comme on pouvait s'y attendre dans un pays aussi polycentré que l'est la Confédération helvétique. Services aux consommateurs et services aux producteurs semblent obéir à des logiques d'implantation différentes. C'est bien ce que confirme l'analyse factorielle de l'emploi tertiaire à l'échelle du système urbain.

\subsection{La distribution des services et la structure du réseau urbain}

D'une première analyse, portant sur les treize variables retenues (cf. figure 6), exprimées en valeur absolue, une seule composante se dégage, représentant à elle seule $87 \%$ de la variance totale: c'est dire que la hiérarchie du réseau urbain tel qu'il est exprimé par ces variables peut être décrite à travers une seule dimension, évidemment médiatisé par les effets de taille. La fig. 7 illustre une classification des villes en cinq niveaux hiérarchiques obtenue au terme d'une analyse de groupement (algorithme de Ward) et en éliminant l'effet de taille (cf. A. CUNHA. J.-B. RACINE, 1984). Cette hiérarchie fonctionnelle est intéréssante pour l'analyse de la répartition des activités tertiaires, car les services orientés vers la population se répartissent en fonction de la dimension des villes, tandis qu'une large proportion des autres services est attirée vers les centres qui remplissent des fonctions internationales, nationales ou cantonales. Zurich et Genève dominent largement les autres unités. Mais ce qui est plus intéressant à souligner est que la hiérarchie fonctionnelle ne correspond pas nécessairement à la hiérarchie dimensionnelle. Genève apparaît en meilleure position que Bâle. Neuchâtel (13 $3^{\text {ème }}$ rang au point de vue dé-

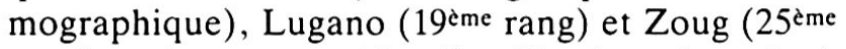
rang) se situent parmi les dix villes les mieux équipées en services. Ces trois centres, avec Lucerne et St. Gall, forment les appuis les plus évidents des «métropoles» du pays. Les autres villes (Baden, Winterthour, Sion, Fribourg, Bienne, Soleure, Aarau, Schaffhouse, Coire, Locarno) rejoignent les centres moyens au niveau quatre parmi lesquelles se trouve la quasi totalité des capitales cantonales. Le niveau cinq voit défiler l'ensemble des autres petites villes. Cela semble confirmer l'hypothèse initiale quant à l'insuffisance des villes moyennes. En effet, seule une partie des villes moyennement grandes et des villes moyennes offrent des prestations de services spécialisés qui correspondent également à leur importance mesurée en nombre d'habitants. 


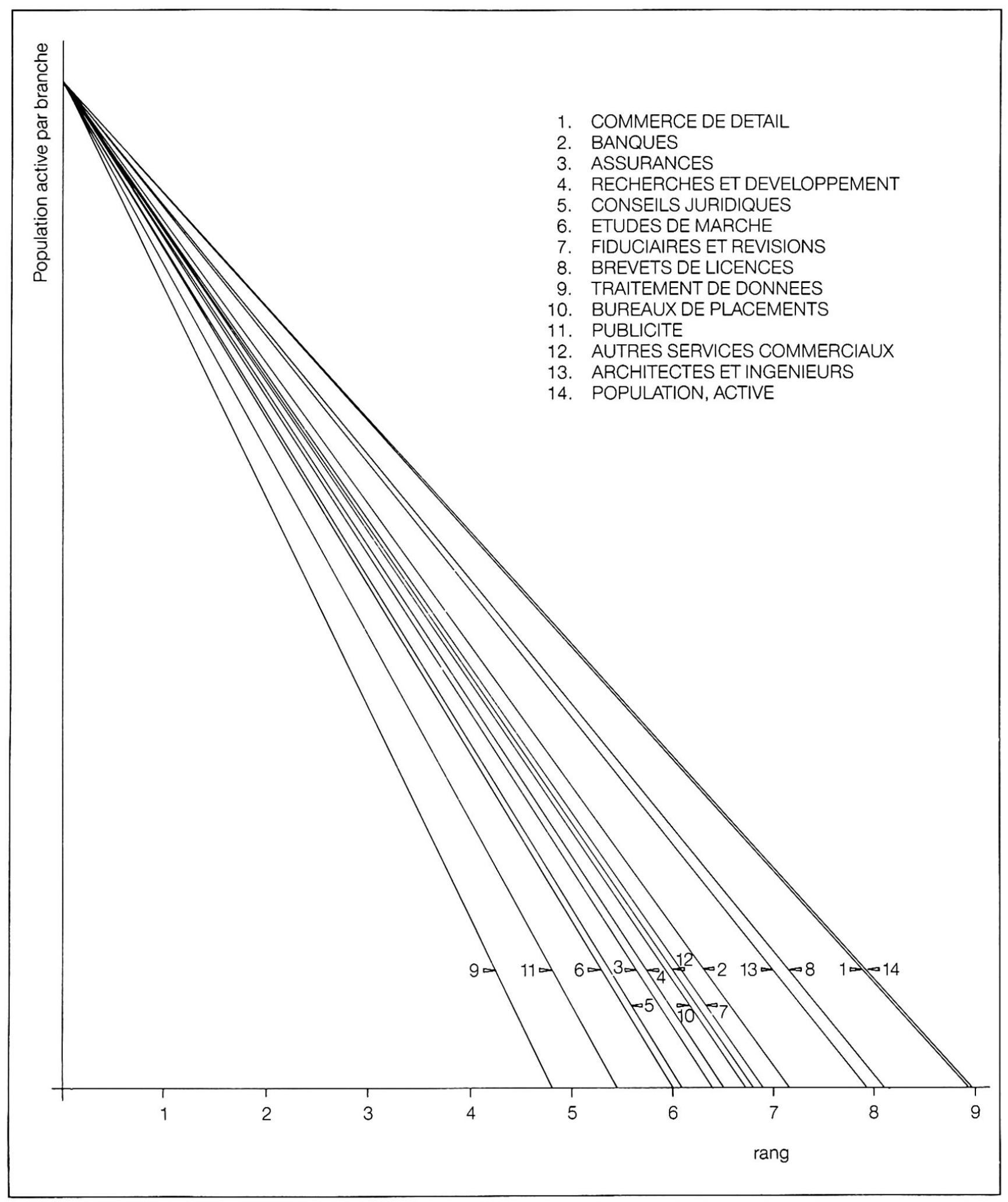

Fig. 6 Pentes des droites de régression selon la rang-taille pour quelques branches des services. 


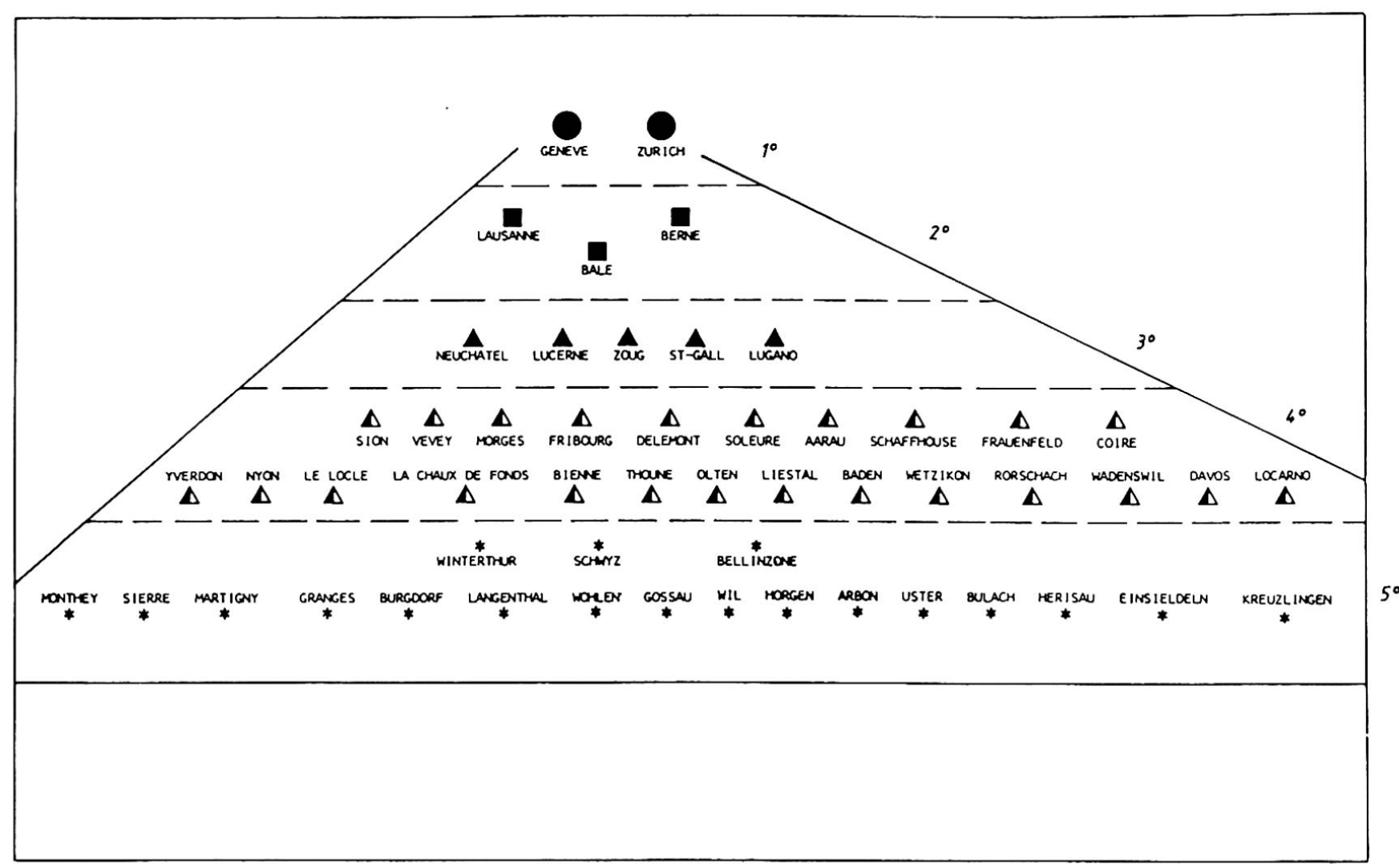

Fig. 7 Classification des villes suisses - sans l'effet de taille - selon la hièrarchie des services, 1975. Source: Cunha, Racine, 1984

L'analyse à l'échelle cantonale et celle à l'échelle du système urbain s'éclairent mutuellement. La genèse du réseau urbain ainsi que la décentralisation politique du pays ont sans doute favorisé une répartition spatiale relativement équilibrée des services sociaux et des fonctions banalisées. En revanche, on peut se demander si cette diffusion relative n'a pas comme contrepartie une concentration plus forte des services très spécialisés dans les niveaux supérieurs de la hiérarchie des lieux centraux. Certaines villes (notamment les villes du pied du Jura) souffrent incontestablement de la domination métropolitaine des plus grands centres géographiquement trop proches. Globalement, le déséquilibre centrepériphérie pourrait avoir tendance à se reproduire dans le temps en raison de la rétention des activités tertiaires de décision et de gestion économique dans les niveaux supérieurs de la hiérarchie des lieux centraux. C'est en tout cas une hypothèse de travail que la publication des données du recensement fédéral des entreprises pour 1985 permettra de confirmer ou d'infirmer.

Quoiqu'il en soit, l'hétérogénéité interne du secteur tertiaire est devenue un fait incontestable et si les dynamiques qui animent la distribution spatiale des divers types d'activité sont elles-mêmes différen- ciées il devient urgent de définir des politiques territoriales adaptées à la nouvelle conjoncture. Comme le souligne, R. RATTI (1985), le fait que l'avenir des régions dépende aujourd'hui de l'intensité des connaissances plus que du travail ou du capital attribue aux activités de services un rôle stratégique, qui peut être saisi par les opérateurs privés ou publics, voire par les deux, en tant qu'instrument de promotion régionale.

\section{En guise de conclusion: pour une autre politique de développement régional}

L'analyse de l'emploi tertiaire non seulement éclaire d'un jour particulier les disparités à l'intérieur du pays, mais encore nous amène à examiner le bien-fondé des politiques de développement régional.

Tant les Cantons que la Confédération sont pris dans un champ de tensions entre deux objectifs économiques, le respect de la liberté du commerce et de l'industrie, impliquant une politique de non-intervention, sinon sur les mécanismes monétaires et conjoncturels de caractère national ou internatio- 
nal, et, à l'opposé, la défense et des régions rurales menacées par le dépeuplement et des cantons peu industrialisés (période 1950 à 70) ou affaiblis par les pertes d'emplois (1970-85).

Si nous considérons tout d'abord la politique régionale au sens restreint, c'est-à-dire l'aide directe aux entreprises ou aux collectivités locales chargées d'investir, la Confédération est plus limitée dans son action que les Cantons, du point de vue de ses compétences constitutionnelles, mais dispose ou a longtemps disposé de moyens financiers supérieurs à la plupart des Cantons. Si nous laissons de côté toute la politique agricole, bien qu'elle présente à l'évidence des aspects de soutien à l'économie des régions défavorisées, les mesures de soutien aux entreprises et aux services sont généralement d'ordre financier (facilités fiscales, subventions, prêts, garanties, etc.), ou pratique (octroi de terrains, conseils, etc.). La Confédération n'a pu, dans une première période s'étendant jusqu'aux débuts des années septante, intervenir que pour des raisons conjoncturelles. Les Cantons, qui disposaient de compétences plus larges, se sont lancés dans une compétition parfois vive pour obtenir des implantations d'entreprises. Depuis 1974, la Confédération a choisi de soutenir les régions de montagne par toute une série de mesures, la plus connue étant les prêts accordés à la réalisation d'équipements destinés à accroître l'attrait des régions de montagne pour les entreprises et les habitants. Il s'agit, cette fois, de mesures indirectes à l'égard des entreprises, comme les Cantons les utilisaient déjà depuis longtemps, notamment pour les constructions de routes ou d'écoles. Enfin, depuis 1978 pour la Confédération et déjà auparavant pour certains Cantons, des aides sont accordées à des entreprises - existantes ou nouvelles - dans le cadre de la lutte contre la crise de cette dernière décennie. A côté des traditionnelles mesures financières ou d'équipement, de nouveaux dispositifs sont mise en place, notamment pour encourager l'innovation.

Quelles branches d'activité ont-elles été touchées par cette politique régionale, «strictu senso»? Essentiellement l'industrie, qui était considérée depuis la fin de la guerre comme l'activité motrice. L'attitude du Valais était typique à cet égard (comme Fribourg d'ailleurs pour mentionner des cantons romands), qui a lutté pendant plus de 20 ans sur le plan économique avant tout pour développer le nombre de ses entreprises industrielles. Le secteur de la construction, dont on admet mieux aujourd'hui le rôle régional, n'a jamais fait l'objet d'une politique globale: tantôt il est bridé par la Confédération, à travers des mesures conjoncturelles (arrêtés sur la surchauffe, par exemple) ou nationalistes (textes limitant la vente de biens-fonds aux personnes domiciliées à l'étranger), tantôt il est soutenu, voire stimulé, par les commandes de travaux publics; bien que les économistes soient plutôt réservés sur le caractère d'entraînement de l'économie par le secteur de la construction, il est frappant de voir comment le Valais - pour reprendre cet exemple - a défendu le maintien d'un nombre élevé de postes de travail dans cette branche.

Et le tertiaire? Les multiples facettes de cette notion apparaissent bien, lorsque l'on examine les politiques régionales, toujours au sens restreint. La Confédération n'a explicitement aidé que les entreprises hôtelières, à travers la Société suisse de crédit hôtelier - si l'on laisse de côté les mesures «d'assainissement» prises dès le début de la première guerre mondiale. Selon une constatation maintes fois répétée depuis 20 ans, l'effet est demeuré mineur, sauf dans quelques cantons dont les autorités fournissent une aide complémentaire.

Les Cantons ont fait plus, puisque plusieurs d'entre eux ont réussi à attirer sur leur territoire non seulement des industries, mais encore des sociétés commerciales ou de services, certaines venant d'autres parties du pays, d'autres de l'étranger; ces implantations avaient d'abord pour but d'accroître la masse fiscale imposable, mais depuis 1975 , elles ont aussi permis de créer des emplois bienvenus. Cette politique, toutefois, ne peut que rarement être considérée comme ayant des effets de correction à l'égard des disparités régionales puisque, à côté d'un canton comme Fribourg, c'est par exemple Zoug qui détient la palme.

Peut-on dire que la politique à l'égard des commerces de détail constitue un effort de redistribution économique régionale? Certes pas; le problème du soutien aux commerçants de localités isolées, spécialement en montagne, n'a pas trouvé de solution légale; quant aux Cantons qui ont cherché à limiter l'explosion des grandes surfaces suburbaines, ils n'ont pas eu un grand succès; le combat, sur ce point, est d'ailleurs à évaluer au niveau non pas du canton ou du pays mais à celui de l'agglomération urbaine, l'enjeu étant surtout la fonction des centres-villes. Reste la loi d'aide aux investissements en région de montagne (LIM), qui, depuis 1974, permet l'octroi de prêts à de nombreux équipements de caractère tertiaire. Or, les cartes et tableaux qui viennent d'être décrits montrent que c'est justement dans le domaine de ces services sociaux et personnels que les cantons dits périphériques sont le mieux dotés. Evidemment, il est heureux que les autorités veillent à maintenir cette bonne dotation, mais celà représente un volet bien secondaire de la politique régionale.

Le nerf du développement régional sur le plan économique, ce sont les services aux entreprises. La demande d'une plus grande efficacité dans la recherche de marchés, dans l'obtention de financement, dans le perfectionnement des fonctions de production, mais aussi la recherche d'une plus grande sou- 
plesse dans l'organisation du travail et dans la gestion des entreprises assurent désormais un rôle essentiel aux activités de services aux entreprises. Au moment où les régions périphériques se soucient, à juste titre, du maintien de leur emploi industriel et ceci pour quasiment toutes les branches et plus seulement pour l'horlogerie ou le textile, il convient non seulement d'appuyer les entreprises industrielles, mais encore de leur fournir l'environnement de services qui leur permette de se maintenir sur le marché international, qui est devenu la norme de réussite.

Nous ne pensons pas que la diffusion des facilités liées aux télécommunications et à la bureautique suffise à repondre à ces besoins. A part certains services très spécialisés, l'entreprise veut avoir un interlocuteur proche et pas seulement à portée de téléphone. MONNOYER et PHILIPPE, lors d'un récent colloque (1986), citaient le cas des régions françaises: au début, Paris et l'Ile-de-France concentraient tous les bureaux de conseil aux entreprises. Ensuite les Chambres de commerce ont commencé à prendre la relève et l'on trouve maintenant dans toutes les grandes villes de province des entreprises spécialisées dans le conseil aux firmes. On ne peut donc que se réjouir de voir se créer en Suisse des entreprises similaires, parfois avec l'aide des pouvoirs pu-

\section{Bibliographie}

BAILLY, A.; MAILLAT, D. (1986): Le secteur tertiaire en question, E.R.E., Genève.

BARCET, A. (1986): Les services dans le système productif. Vers une typologie de la production de services. Colloque "Les services et le développement économique: vers une nouvelle typologie des activités de service", Genève.

BELL, D. (1973): Vers la société post-industrielle, Laffont, Paris.

BROWNING, H.; SINGELMANN (1978): The Transformation of the US Labour Force: the interaction of industry and occupation. In: Politics and Society, pp. 481-509.

CLARK, C. (1960): Les conditions du progrès économique. PUF, Paris.

CUNHA, A.; RACINE, J.-B. (1984): Le rôle des services aux entreprises dans une société post-industrielle: technologies nouvelles et décentralisation. In: Revue d'économie régionale et urbaine, no. 5, pp. 731-756.

DEISS, J. (1985): Diagnostic et perspectives du tertiaire fribougeois. In: Le secteur tertiaire et le nouveau développement régional, Eds. Universitaires, Fribourg.

FISCHER, A. (1947): Progrès économique et sécurité sociale, Médicis, Paris. blics comme - pour prendre des exemples romands - La RETà La Chaux-de-Fonds ou la SODEVAL en Valais.

Toutefois les objectifs de politique régionale ne peuvent que partiellement être atteints par les mesures de soutien, directes ou indirectes, aux entreprises. Comme les chercheurs et les experts du Programme national «Région» l'ont bien montré (R. FREY, 1985), il faut pousser l'analyse au-delà du constat des handicaps frappant les régions de montagne et de vieille industrialisation et proposer des mesures beaucoup plus globales. D'une part, il convient d'aborder la question des relations inégales au sein des agglomérations urbaines et d'autre part il faut replacer les mesures économiques dans leur cadre politique plus général, celui du partage des compétences entre les diverses entités politiques, entre les communes d'une même région ou d'une même agglomération et entre les cantons. La péréquation entre les communes dans un canton et entre les cantons doit être renforcée; de même les instruments de redistribution des revenus entre couches sociales sont des instruments essentiels d'un meilleur équilibre entre toutes les parties du pays. La répartition des services pourrait être l'indice d'un développement régional plus équilibré et plus autonome, à condition de prendre en compte toutes les facettes de ces activités.

FREY, R. (1985): Politique régionale, une évaluation, Presses Polytechniques Romandes, Lausanne.

FUCHS, V. (1968): The Service Economy, Columbia University Press, New York.

GEORGE, P. (1978): Populations actives: introduction à une géographie des populations, PUF, Paris.

HAUTREUX, J.; ROCHEFORT, M. (1964): La fonction régionale dans l'armature française, CNAT, Paris.

LENGELLE (1966): La révolution tertiaire, Génin, Paris.

MONNOYER, M.-C.; PHILIPPE, J. (1986): L'importance des services aux entreprises pour le développement économique. Colloque Les services et le développement économique: vers une nouvelle typologie des activités de service, $\mathrm{Ge}$ nève.

OCDE (1977): La politique de l'innovation dans le secteur des services, OCDE, Paris.

RATTI, R. (1985): Les régions suisses de 1985 face aux problèmes du tertiaire. In: Le secteur tertaire et le nouveau développement régional, Eds. Universitaires, Fribourg.

ROCHEFORT, M. (1976): Les activités tertiaires. Leur rôle dans l'organisation de l'espace, CDU \& SEDES, Paris.

SCRIS (1982): Le secteur tertiaire dans le Canton de Vaud et en Suisse, SCRIS, Lausanne. 